\title{
Patient Safety in Inpatient Wards: Sample of a University Hospital
}

\author{
${ }^{1}$ Fereshteh Farzianpour, ${ }^{2}$ Abbas Rahimi Fouroshani, \\ ${ }^{3}$ Kiyana Ragi Dargaha and ${ }^{4}$ Seyed Shahab Hosseini \\ ${ }^{1}$ Department of Health Management and Economics, \\ ${ }^{2}$ Department of Epidemiology and Statistic, \\ School of Public Health Tehran, University of Medical Sciences, Iran \\ ${ }^{3}$ Department of Health Management and Economic, School of Management, Azad University, Tehran, Iran \\ ${ }^{4}$ Department of Management, Tehran University, International Campus-Kish Island, Iran
}

Received 2012-08-27, Revised 2012-12-28; Accepted 2012-12-29

\begin{abstract}
The aim of this study was to evaluate the patient safety in inpatient wards of an educational hospital affiliated with Tehran University of Medical Sciences from the viewpoint of nursing staff using the six sigma methodology. This was a cross-sectional descriptive analytical study, carried out in an educational hospital affiliated with Tehran University of Medical Sciences in 2010. The study was performed using a researcher-devised questionnaire. From among the hospital nurses, 42 nurses (three from each ward) were randomly selected and were asked to fill out the questionnaire. We used the opinions of faculty members and experts of the field to determine content validity of the questionnaire. Furthermore, to confirm the questionnaire reliability, the value of Cronbach's alpha was calculated and it was determined as 0.81 . Data analysis was performed using SPSS and Microsoft Excel software. According to the results, the levels of physical environment and installation safety and safety training were medium $(59.8,60.2$ and $64.6 \%$, respectively), while safety of patients' beds, health and management of incidents were at desirable level (70.6, 76.6 and $77.2 \%$, respectively). In general, safety of inpatient wards of the hospital was at the medium level. From the view point of nursing staff, the wards Urology 2 and Orthopedic Surgery 1 had the best and worst status with the mean score of 91.23 and 58.52 , respectively.
\end{abstract}

Keywords: Safety, Six Sigma Model, Nursing Staff, Teaching Hospital

\section{INTRODUCTION}

Hospitals are among the most important health care providing organizations (Farzianpour et al., 2011a). Hospital have sophisticated and advanced facilities and instruments and specialized and semi-specialized workforce to provide, maintain and support one of the basic needs of human (Nishizaki et al., 2010). Before providing any services, hospitals should provide an appropriate space and safe environment for their clients. Moreover, hospitals should have facilities and equipment required for dealing with emergency conditions, since irreparable physical and human loss may occur due to unexpected events (Stone et al., 2007).

Before dealing with their responsibilities, level of safety in hospitals should be evaluated and some measures be adopted to improve the safety levels (JC, 2011). This is because although patients should receive care in hospitals, hospitals are responsible for the referred individuals and visitors and incidents should be controlled (JCI, 2011).

Safety in health care organizations is a set of measures adopted for protection of physical assets of the organization and the individuals interact with the

Corresponding Author: Fereshteh Farzianpour, Department of Health Management and Economics, School of Public Health Tehran University of Medical Sciences, Iran 
organization and its surrounding environment (Rogers et al., 2004).

Protection and safety are adopted to reduce the risk of harms and loss and does not exclusively eliminate the risks (Decker, 2012; Greenwald et al., 2010). Safety is not a static concept and is always variable. What seems safe today may not be safe tomorrow. Safety is a phenomenon, which requires periodical evaluations. Presence of a safety plan in hospitals is an important item. Safety plans may save people's life, prevent the harms and finally reduce the hospital costs. Design and employment of safety plans in hospitals is an effective and appropriate task (JCAH, 2011). In fact, safety is a word, which is effective and real just when it is accompanied by specialized programming, checking, presence of safety plan, training and exercise of dealing with incidence, continuous goal-oriented health programs and required trainings (Coughlin et al., 2012).

In the recent decade, the six sigma model has received attention as a robust systematic approach in improvement of health care services, reduction of the costs, improvement of patient safety, increasing the efficiency of resources and overcoming the challenges (Koning et al., 2006; Bisgaard and Freiesleben, 2004).

Considering the effectiveness of the six sigma method in reducing pitfalls of the health care system and the importance of patient safety and since patient safety is a major concern in the health care system, the authors attempted to evaluate patient safety level in inpatients wards of the university Hospital using the six sigma models.

\section{MATERIALS AND METHODS}

This is a cross-sectional descriptive analytical study, carried out in an educational hospital affiliated with Tehran University of Medical Sciences in 2009. A questionnaire was prepared by the author according to literature review and the guides provided by faculty members and research consultant of the Department of Health Management. Then, after obtaining the required permissions from the hospital manager and educational supervisor, the questionnaires were distributed among the nurses. The hospital has 14 wards and we randomly selected three nurses from each ward; thus, 42 questionnaires were handed in and one day later the participants were asked to return completed questionnaires. To determine the questionnaire validity, the questionnaire was reviewed by some faculty members and they confirmed its validity. To confirm the questionnaire reliability, the value of Cronbach's alpha was determined as 0.81 using SPSS software. The data was analyzed using SPSS and Microsoft Excel software.
Firstly, the items were scored on a five-point Likert scale (none/never/no, few/once in a while, some/sometimes, most/most of the time, all/always/yes). Then, the items of the questionnaire were classified into the following six categories; safety of physical environment, safety of patients' beds, installations, health, safety training and management of incidents. After summing up the scores and calculation of mean and mean percentage for each ward and the hospital in general, scores above 70, 40-70 and below 30 were considered as desirable, medium and poor, respectively. Then, the standard deviation, Upper Specification Limit (USL) and Lower Specification Limit (LSL) values for each category of items in each ward and all the wards were calculated. In the following, using the six sigma method, the wards with the specification interval larger than that calculated for the hospital was specified. The reason for this larger interval was the higher dispersion of the responses.

\section{RESULTS}

We evaluated 14 wards and in each ward, three nurses were randomly selected and were asked to fill out the questionnaire on patient safety in inpatient wards of the hospital. From among the nurses participated in the study, $33.3 \%$ were supervisors and $97.6 \%$ of the participants were female. With regard to the age group, the highest $(11.9 \%)$ and lowest $(2.4 \%)$ frequency was observed in 36-38 and below 23 age groups, respectively. In addition, all participants had B. Sc. of nursing.

The findings of the study for each ward were as follows.

\subsection{CCU Ward}

In this ward, safety of patients' beds, installations, health, safety training and management of incidents were in the desirable level, while safety of physical environment was medium.

\subsection{Men's Internal Medicine Ward}

Health and management of incidents in this ward were in the desirable level, while safety of physical environment, installations and safety training were in the medium level.

\subsection{Women's Internal Medicine Ward}

In this ward, safety of physical environment, health, safety training and management of incidents were in the desirable level and safety of patients' beds and installations were in the medium level. 


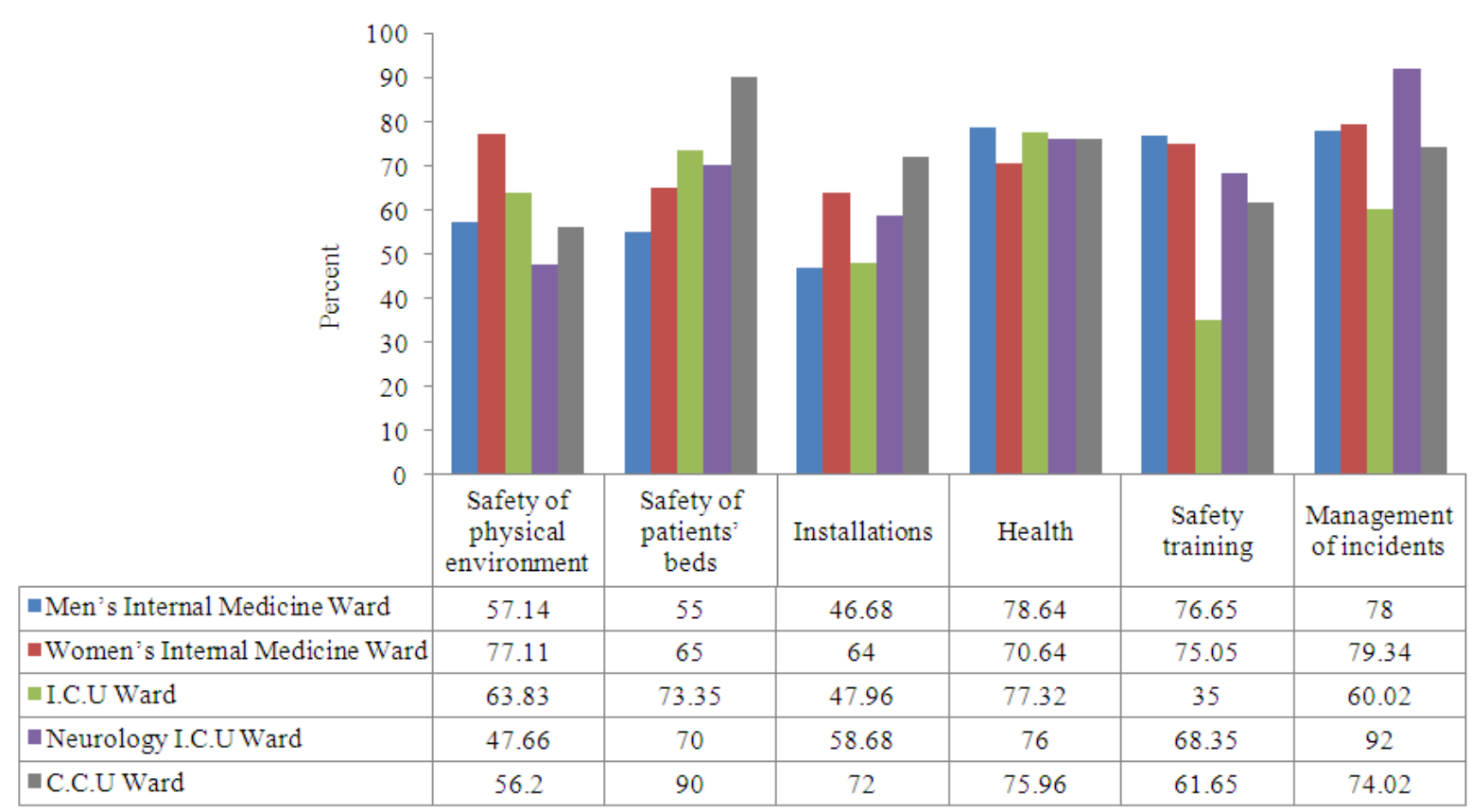

Fig. 1. Safety status inpatient wards, a teaching hospital in the Tehran University of Medical Sciences-2010

\subsection{ICU Ward}

Safety of patients' beds, health and safety training were desirable, while safety of physical environment, installations and management of incidents were in the medium level in this ward.

\subsection{Neurology ICU Ward}

Considering safety of patients' beds, health, safety training and management of incidents, the ward was in a desirable state, while safety of physical environment and installations were in medium level (Fig. 1).

\subsection{Men's Neurosurgery Ward}

In this ward, health, safety training and management of incidents were in the desirable level; and safety of physical environment, safety of patients' beds and installations were in medium level.

In this ward, the USL-LSL interval for all categories of items lied within the normal range of the hospital.

\subsection{Women's Neurosurgery Ward}

In this ward, safety of patients' beds, health, safety training and management of incidents were in the desirable level; and safety of physical environment and installations were in the medium level.

\subsection{Men's Surgery Ward}

In this ward, safety of patients' beds, safety training and management of incidents were in the desirable level, while safety of physical environment, installations and health were in the medium level.

\subsection{Urology Ward 1}

Level of safety in this ward was found to be desirable with regard to safety of patients' beds, health, safety training and management of incidents, while safety of the physical environment and installations were found to be medium.

\subsection{Urology Ward 2}

Considering safety of physical environment, safety of patients' beds, installations, health, safety training and management of incidents, the ward had a desirable safety level.

\subsection{Men's Neurology Ward}


Safety of patients' beds, health, safety training and while safety of physical environment and installations management of incidents were in the desirable level, were medium.

Table 1. Safety Status inpatient wards, a teaching hospital in the Tehran university of medical sciences-2010

\begin{tabular}{|c|c|c|c|c|c|}
\hline Dimensions safety wards & Mean & Percentage of mean & SD & USL & LSL \\
\hline 1-Neurosurgery men & & & & & \\
\hline SPE & 2.71 & 54.29 & 1.31 & 6.64 & --1.49 \\
\hline SPB & 3.34 & 66.70 & 1.61 & 8.17 & -2.81 \\
\hline I & 3.20 & 64.00 & 2.01 & 9.23 & -1.51 \\
\hline $\mathrm{H}$ & 2.87 & 57.36 & 1.46 & 7.25 & -1.16 \\
\hline ST & 2.08 & 41.65 & 1.08 & 5.32 & 0.78 \\
\hline MI & 3.94 & 78.64 & 1.05 & 7.08 & 1.22 \\
\hline \multicolumn{6}{|l|}{ 2-Neurosurgery women } \\
\hline SPE & 2.79 & 49.49 & 1.44 & 6.79 & -1.85 \\
\hline SPB & 3.50 & 70.00 & 1.73 & 8.69 & -1.69 \\
\hline I & 2.60 & 52.00 & 2.03 & 8.69 & -3.49 \\
\hline $\mathrm{H}$ & 4.80 & 96.00 & 0.56 & 6.64 & 3.12 \\
\hline ST & 2.00 & 41.65 & 1.78 & 7.42 & -3.26 \\
\hline MI & 4.30 & 86.02 & 0.99 & 7.27 & 1.35 \\
\hline \multicolumn{6}{|l|}{ 3-General surgery women } \\
\hline SPE & 2.71 & 54.29 & 1.31 & 6.64 & -1.22 \\
\hline SPB & 3.34 & 66.70 & 1.61 & 8.17 & -1.49 \\
\hline I & 3.20 & 64.00 & 2.01 & 9.23 & -2.81 \\
\hline $\mathrm{H}$ & 2.87 & 57.36 & 1.46 & 7.25 & -1.51 \\
\hline ST & 2.08 & 41.65 & 1.08 & 5.32 & -1.16 \\
\hline \multirow{2}{*}{\multicolumn{6}{|c|}{ 4-urethra 1}} \\
\hline & & & & & \\
\hline SPE & 3.09 & 61.89 & 1.64 & 8.01 & -1.83 \\
\hline SPB & 3.83 & 76.65 & 1.8 & 9.23 & -1.57 \\
\hline I & 2.33 & 46.68 & 1.95 & 8.18 & -3.52 \\
\hline $\mathrm{H}$ & 3.80 & 75.96 & 1.57 & 8.51 & -0.91 \\
\hline ST & 4.25 & 85.00 & 1.14 & 7.67 & 0.83 \\
\hline MI & 4.10 & 82.00 & 0.88 & 6.74 & 1.46 \\
\hline \multicolumn{6}{|l|}{ 5-urethra 2} \\
\hline SPE & 3.95 & 79.03 & 0.67 & 5.96 & 1.94 \\
\hline SPB & 4.92 & 98.35 & 0.29 & 5.79 & 4.05 \\
\hline I & 4.13 & 82.68 & 1.64 & 9.05 & -0.79 \\
\hline $\mathrm{H}$ & 5.00 & 100.0 & 0.00 & 5.00 & 5.00 \\
\hline ST & 5.00 & 100.0 & 0.00 & 5.00 & 5.00 \\
\hline MI & 4.37 & 87.34 & 1.25 & 8.12 & 0.62 \\
\hline \multicolumn{6}{|l|}{ 6-Safety neurology men } \\
\hline SPE & 2.76 & 55.23 & 1.55 & 7.41 & -1.89 \\
\hline SPB & 3.50 & 70.00 & 1.73 & 8.69 & -1.69 \\
\hline I & 3.40 & 68.00 & 2.03 & 9.49 & -2.69 \\
\hline $\mathrm{H}$ & 4.60 & 92.04 & 1.06 & 7.78 & 1.42 \\
\hline ST & 3.83 & 76.60 & 1.40 & 8.03 & -0.37 \\
\hline MI & 4.00 & 80.00 & 1.26 & 7.78 & 0.22 \\
\hline \multicolumn{6}{|l|}{ 7-Neurology women } \\
\hline SPE & 3.19 & 63.83 & 1.47 & 7.6 & -1.22 \\
\hline SPB & 3.33 & 66.65 & 1.67 & 8.34 & -1.68 \\
\hline I & 2.73 & 54.68 & 1.79 & 8.1 & -2.64 \\
\hline $\mathrm{H}$ & 3.07 & 61.32 & 1.62 & 7.93 & -1.79 \\
\hline ST & 3.00 & 60.00 & 1.28 & 6.84 & -0.84 \\
\hline \multirow{2}{*}{\multicolumn{6}{|c|}{ 8-Orthopedics1 }} \\
\hline & & & & & \\
\hline SPE & 2.24 & 44.77 & 1.37 & 6.36 & -1.88 \\
\hline SPB & 3.09 & 61.70 & 1.88 & 8.73 & -2.55 \\
\hline I & 2.80 & 56.00 & 2.01 & 8.82 & -3.22 \\
\hline $\mathrm{H}$ & 3.60 & 72.00 & 1.55 & 8.25 & -1.05 \\
\hline ST & 2.17 & 43.35 & 1.27 & 5.97 & -1.63 \\
\hline MI & 3.67 & 73.32 & 1.35 & 7.71 & -0.37 \\
\hline \multicolumn{6}{|l|}{ 9-Orthopedics2 } \\
\hline SPE & 3.33 & 66.66 & 1.28 & 7.17 & -0.51 \\
\hline SPB & 3.33 & 66.66 & 1.67 & 8.34 & -1.68 \\
\hline I & 2.73 & 54.64 & 1.98 & 8.67 & -3.21 \\
\hline $\mathrm{H}$ & 3.27 & 65.36 & 1.49 & 6.73 & -2.19 \\
\hline ST & 2.92 & 58.35 & 1.24 & 6.64 & -0.80 \\
\hline Science Publications & & 2007 & & & AJAS \\
\hline
\end{tabular}


Safety of physical environment $=$ SPE Safety of patients' beds $=$ SPB Installations $=$ I Health $=$ H Safety training $=$ ST Management of incidents $=$ MI

Table 2. Mean and mean percent patient safety in inpatient wards of a University Hospital; Viewpoints of Nursing Staff, 2010

\begin{tabular}{|c|c|c|c|c|c|}
\hline $\begin{array}{l}\text { Dimension of } \\
\text { safety total wards }\end{array}$ & M & Percentage of $\mathrm{M}$ & SD & USL & LSL \\
\hline $\begin{array}{l}\text { Safety of physical } \\
\text { environment }\end{array}$ & 2.99 & 59.8 & 1.49 & 7.45 & -1.48 \\
\hline Safety of patients' beds & 3.53 & 70.6 & 1.64 & 8.46 & -1.40 \\
\hline Installations & 3.01 & 60.2 & 1.94 & 8.83 & -2.81 \\
\hline Health & 3.83 & 76.6 & 1.50 & 8.34 & -0.67 \\
\hline Safety training & 3.23 & 64.4 & 1.59 & 8.00 & -1.55 \\
\hline Management of incidents & 3.86 & 77.2 & 1.18 & 7.41 & 0.31 \\
\hline
\end{tabular}

\subsection{Women's Neurology Ward}

Considering all the items of safety of physical environment, safety of patients' beds, installations, health, safety training and management of incidents, the ward was medium.

\subsection{Orthopedic Surgery Ward 1}

Level of safety of the ward with regard to health and management of incidents was desirable, while it was medium for safety of physical environment, safety of patients' beds, installations and safety training.

\subsection{Orthopedic Surgery Ward 2}

Safety level of the ward considering safety training and management of incidents was desirable, while safety of physical environment, safety of patients' beds, installations and health were.

Three standard deviations on either side of the mean in all inpatient wards were -1.48 to $7.45,-1.40$ to 8.46 , 2.81 to $8.83,-0.67$ to $8.34,-1.55$ to 8 and 0.31 to 7.41 for safety of physical environment, safety of patients' beds, installations, health, safety training and management of incidents, respectively.

The specification interval larger than that calculated for the hospital indicates the dispersion of the responses of the participants.

The USL-LSL interval of safety of physical environment, safety of patients' beds, installations, health, safety training and management of incidents for the inpatient wards, which were larger than those of the hospital are provided in the following:

- Safety of physical environment: ICU, Urology 1 and Men's Neurology wards

- Safety of patients' beds: Women's Internal Medicine, ICU, Neurology ICU, Women's
Neurosurgery, Urology 1, Men's Neurology Women's Neurology, Orthopedic Surgery 1 and Orthopedic Surgery 2 wards

- Installations: ICU, Women's Neurosurgery, Men's Surgery, Urology 1, Men's Neurology, Orthopedic Surgery 1 and Orthopedic Surgery 2 wards

- Health: Women's Internal Medicine, ICU, Urology 1, Women's Neurology and Orthopedic Surgery 1 wards

- Safety training: Neurology ICU, Urology 1, Women's Neurology and Orthopedic Surgery 1 wards

- Management of incidents: ICU, Urology 2, Men's Neurology and Orthopedic Surgery 1 (Table 1 and 2)

\section{DISCUSSION}

In this study, patient safety in hospitals was defined in six categories of safety of physical environment, safety of patients' beds, installations, health, safety training and management of incidents. Then, using the questionnaire, measurements were carried out according to the definitions. The results were as follows.

With regard to safety of physical environment, the inpatient wards were medium with the mean score of $59.8 \%$ and only the Women's Internal Medicine and Urology 2 wards were in a desirable state.

The highest and lowest mean scores for safety of physical environment were 79 and $44.77 \%$, which were respectively obtained for Urology 1 and Orthopedic Surgery wards.

In our literature review, we could not find papers on evaluation of hospital safety using the six sigma model. Thus, we could not compare our results with similar studies. However, we elaborate on the factors interfering with the safety and also how to develop safety in this educational hospital. 
Lack of window guards, inappropriate flooring, not using isolated rooms when required and lack of air conditioning system are the factors that affect patient safety in these wards. All windows in inpatient wards should be equipped with guards and the flooring should be waterproof to provide patient safety and comfort (Cunningham et al., 2012; Farzianpour et al., 2011b).

Flooring of patients' rooms and ward corridors should be made from non-slip materials. Synthetic materials are preferred for this purpose (Neuhausen et al., 2012; JCI, 2011).

Flooring of the baths should be made from specific materials, such that nurses could have enough control over the patients. Covering of the stairways should always be kept clean and be made from non-slip materials and also minimize the noise as much as possible (Greenwald et al., 2010; JCI, 2011).

The buildings should regularly be checked from cracks.

The walls in all wards should be normal, without any cracks and be in light colors and be washable up to the height of $1.8 \mathrm{~m}$. The ceilings in all parts of the hospital should be smooth and without cracks and be painted in light colors.

Each ward should have appropriate full-time ventilation to provide clean air with proper temperature and a comfortable space for the patients (JCAH, 2011).

Isolation of patients is performed to separate the patients from other individuals and nurses. In fact, this is a cautious measure to prevent dissemination and intraward spread of infectious pathogens among patients, staff and visitors of the wards.

Ventilation is considered as a technique for disinfection of the environment. The roles of the ventilation system in hospitals are maintaining the temperature of inner spaces and reduction of microbial load, dusts and smells in the air. The ventilation systems should be designed such that appropriate setting of the inward and outward air flow volume maintains the air flow in the desirable direction.

With regard to the safety of patients' beds, the results indicated that the mean score was $70.6 \%$ and it was in a desirable level.

The best and worst results for safety of patients' beds were obtained for the Urology 2 and Men's Internal Medicine wards, with the mean scores of $98.35 \%$ and $55 \%$, respectively.

Lack of bedside nurse call system in most wards and absence of footstep beside the beds in some wards may endanger the patients.

An option for patients' beds is the possibility of attaching the bedside safety rails to avoid falls
(Rogers et al., 2004). Falls from hospital beds is the major cause of injury of patients, especially in older age groups; such that more than $70 \%$ of victims of the falls leading to death are patients above 65. Falls from hospital beds is apparently the most frequent and troublesome accident. Physical control (tying arms and legs) is not desirable and welcomed in most cases. Furthermore, 24-h direct observation and nursing except for few cases is very costly and impossible. Therefore, such problems should be separately analyzed for each patient and decisions about the approach should be made according to the case characteristics.

At the bedside of all patients, an appropriate communication tool (such as phone for external and nurse call button for internal communications) should be available.

Hospital footstep (single step at the bedside) is a basic equipment of inpatient wards (Leibrock and Harris, 2011; JCAH, 2011).

With regard to installations, the results indicated that installations of the inpatient wards was medium with the mean score of $60.2 \%$ and only the three wards of CCU, Men's Neurosurgery and Urology 2 were in a desirable state in this respect.

The highest score of installations was obtained for the Urology 2 ward, with the mean score of $82.68 \%$, while the lowest score was obtained for Men's Internal Medicine and Urology 2 wards with the mean score of $46.68 \%$.

The improper status of half of electrical sockets and lack of emergency power supply in most wards and not having the possibility of taking emergency exit steps would cause some risks for patients.

All electrical equipment such as sockets and switches should be installed according to safety regulations with protective earth contact. Emergency power supply and energy facilities should be installed in the nurses' station of the wards (Aspden et al., 2004).

Emergency power supply is necessary for hospitals. After failure of central electrical system, the emergency power supply should be automatically started maximally within $10 \mathrm{sec}$.

Fire extinguishing systems should be applied properly and proportionate to the range of activities of the ward.

Fire accidents are caused by severe burning of flammable materials, either intentionally or unintentionally. The accidents harm men, buildings, instruments and facilities. Considering the potential of fire accidents in hospitals and presence of different flammable materials in hospital wards, preventive measures and predictions should be adopted in hospitals. 
For each ward, exit facilities (escape steps) should separately be applied for unpredictable events.

In crises, emergency exits and standard stairways, which were built according to the national regulations of buildings, would play an important role in safety of individuals and timely evacuation of the buildings (Verni, 2012).

To avoid falls of people, the staircases should have appropriate support and fences and stable shelters with suitable height. Moreover, other details considered in the national regulations of buildings should be completely taken into account when designing hospital buildings.

Regarding the health status, the inpatient wards were in a desirable state with the mean score of $76.6 \%$ and only the three wards of Men's Surgery, Women's Neurology and Orthopedic Surgery 2 were medium in this respect.

The best and worst wards with regard to health scores were Urology 2 and Women's Neurology with the mean scores of 100 and $61.3 \%$, respectively.

Absence of garbage chute system, washing and disinfection of bedpans and urinals and irregular collection of garbage in some wards is dangerous for patients owing to the risk of infection.

The floor of all rooms and corridors should be cleaned and disinfected regularly on a daily basis (Leibrock and Harris, 2011; JCAH, 2011).

The facilities required for automatic evacuation, washing and disinfection of the bedpans and urinals without requiring manual efforts should be applied. Washing and disinfection of bedpan.

Proportionate to the increase in admission of inpatient and outpatient cases, hospital wastes would increase. Although the effect of hospital wastes on safety and health is not measurable, without proper management, the infectious wastes lead to contamination of materials, furniture, instruments, patients and staff. Therefore, this is considered a very hazardous source for patients, health care providers and visitors (Woods, 2010).

Hospital infections have negative impacts on patients and the hospital. Because of hospital infections, the patients would stay longer in hospitals. This leads to higher hospital costs. Furthermore, due to losing of qualitative effectiveness of hospital beds, the hospitals would experience loss.

Considering the safety training, the results indicated that the wards were medium with the mean score of $64.4 \%$.
The best ward in this respect was Urology 2 with the mean score of $100 \%$, while ICU was the worst with the mean score of $35 \%$.

Lack of training for using fire extinguishers and lack of safety training for staff endanger patients in some wards, owing to not following the safety regulations by staff.

All staff should be trained for reporting of incidents to those in charge.

The role of training as the foundation of progress and evolution in different aspects of reducing the incidents is of great importance.

Analyzing the statistics of incidence demonstrate that the rate of incidents by staff not having adequate safety training was $25 \%$ higher than that by their trained colleagues.

Safety training addresses three main topics and the courses on safety should include these three items (Ross et al., 2011; Leibrock and Harris, 2011; JCAH, 2011):

- Improvement of knowledge of individuals about the significance of safety and health in development of the country. In other words, the individuals should be thoroughly familiar with theoretical and practical methods of avoiding occupational incidents that are expected and consider "safety first, then work"

- Enhancement of knowledge of individuals about the abilities of preventing events; and

- Improvement of individuals' skills in employment of safety systems and equipment during their work

\section{CONCLUSION}

All health centers should set up continuous programs on patient safety and train their staff in this regard to make safety as a culture among the staff. The programs should be designed such that they can identify the system problems and the underlying causes.

A program on hospital incidents would be successful only if staff members completely participate in it.

Safety training programs should be delivered to the patients and their family.

Considering management of incidents, our results demonstrated that the wards were in a desirable state with the mean score of $77.2 \%$.

The highest and lowest scores on management of incidents were obtained for Neurology ICU and Women's Neurology with the mean scores of 92 and $60 \%$, respectively. The score on putting forward suggestions by the staff was medium. This is while their 
suggestions could minimize the future risk of incidences and even eliminate it.

The studies have shown that the events in general did not have a single underlying cause and they resulted from technical and human reasons. The causes depend on the type, environment, conditions of the working environment and the tools and could be categorized into two types of direct and indirect causes.

The main goal for evaluation of an incidence is collection of information required for defining the principles required for prevention of similar events.

Another measure for modification of individuals and systems is punishment of people who were guilty and abdicate their legal and human responsibilities. The punishment should be used as a tool to prevent repeating a non-feasible and illegal action, such that it modifies the individuals' behavior and the person can play an effective role. A nurse is responsible for the nursing actions and judgments that are performed in relation to individuals. Taking responsibility has been determined as a promotion factor for health care staff, both as a personal factor and as a factor related to the performance. Therefore, following the above-mentioned items would enhance the safety of patients and the hospital.

\section{REFERENCES}

Aspden, P., J.M. Corrigan, J. Wolcott and S.M. Erickson, 2004. Patient Safety: Achieving a New Standard for Care. 1st Edn., National Academies Press, Washington, D.C., ISBN-10: 0309529328, pp: 501.

Bisgaard, S. and J. Freiesleben, 2004. Six Sigma and the bottom line. Q. Progress, 37: 57-62.

Coughlin, T.A., S.K. Long, E. Sheen and J. Tolbert, 2012. How five leading safety-net hospitals are preparing for the challenges and opportunities of health care reform. Health Aff., 31: 1690-1697. DOI: $10.1377 /$ hlthaff.2012.0258

Cunningham, P., L. Felland and L. Stark, 2012. SafetyNet providers in some us communities have increasingly embraced coordinated care models. Health Aff., 31: 1698-1707. DOI: 10.1377/hlthaff.2011.1270

Decker, S.L., 2012. In 2011 Nearly one-third of physicians said they would not accept new Medicaid patients, but rising fees may help. Health Aff., 31: 1673-1679. DOI: 10.1377/hlthaff.2012.0294

Farzianpour, F., M. Arab, S. Amoozagar, A.R. Fouroshani and A. Rashidian et al., 2011a. Evaluation of international standards of Quality
Improvement and Patient Safety (QPS) in Hospitals of Tehran University of Medical Sciences (TUMS) from the managers' point of view. World Applied Sci. J., 15: 647-653.

Farzianpour, F., S. Aghababa, B. Delgoshaei and M. Haghgoo, 2011b. Performance evaluation a teaching hospital affiliated to Tehran University of Medical Sciences Based on Baldrige excellence model. Am. J. Econ. Bus. Admin., 3: 272-276. DOI: 10.3844/ajebasp.2011.272.276

Greenwald, J.L., L. Halasyamani, J. Greene, C. LaCivita and E. Stucky et al., 2010. Making inpatient medication reconciliation patient centered, clinically relevant and implementable: A consensus statement on key principles and necessary first steps. J. Hospital Med., 5: 477-485. DOI: 10.1002/jhm.849

JC, 2011. Improving America's hospitals-the joint Commission's annual report on quality and safety. The Joint Commission.

JCAH, 2011. Hospital Accreditation Standards 2012: Standards, Elements of Performance, Scoring, Accreditation Policies. 1st Edn., Joint Commission Resources, USA., ISBN-10: 1599404257.

JCI, 2011. WHO collaborating centre for patient safety solutions. Joint Commission International.

Koning, H.D., J.P.S. Verver, J.V.D. Heuvel, S. Bisgaard and R.J.M.M. Does, 2006. Lean six sigma in healthcare. J. Healthcare Q., 28: 4-11. DOI: 10.1111/j.1945-1474.2006.tb00596.x

Leibrock, C.A. and D.D. Harris, 2011. Design Details for Health: Making the Most of Design's Healing Potential. 2nd Edn., John Wiley and Sons, New York, ISBN-10: 0470926848, pp: 320.

Neuhausen, K., K. Grumbach, A. Bazemore and R.L. Phillips, 2012. Integrating community health centers into organized delivery systems can improve access to subspecialty care. Health Aff., 31: 1708-1716. DOI: $10.1377 /$ hlthaff.2011.1261

Nishizaki, Y., Y. Tokuda, E.S. Sato, K. Kato and A. Matsumoto et al., 2010. Relationship between nursing workloads and patient safety Incidents. J. Multidisciplinary Healthcare, 3: 49-54. DOI: 10.2147/JMDH.S9699

Rogers, A.E., W.T. Hwang, L.D. Scott, L.H. Aiken and D.F. Dinges, 2004. The working hours of hospital staff nurses and patient safety. Health, Aff., 23: 4202-212. DOI: 10.1377/hlthaff.23.4.202

Ross, J.S., S.M. Bernheim, Z. Lin, E.E. Drye and J. Chen et al., 2011. Based on key measures, care quality for medicare enrollees at safety-net and non-safety-net hospitals was almost equal. Health Aff., 31: 17391748. DOI: $10.1377 /$ hlthaff.2011.1028 
Stone, P.W., C. Mooney-Kane, E.L. Larson, T. Horan and L.G. Glance et al., 2007. Nurse working conditions and patient safety outcomes. Med. Care, 45: 571-578. PMID: 17515785

Verni, C., 2012. A Hospital system's response to a hurricane offers lessons, including the need for mandatory interfacility drills. Health Aff., 31: 18141821. DOI: $10.1377 /$ hlthaff.2012.0154

Woods, M.S., 2010. Effective handoff communication Part 2: Standardizing processes throughout your organization. Joint Commission Perspectives Patient Safety, 10: 3-5. 